Proceedings of the Edinburgh Mathematical Society (2005) 48, 213-218 (C)

DOI:10.1017/S0013091503000798 Printed in the United Kingdom

\title{
APPLICATIONS OF DIVERGENCE POINTS TO LOCAL DIMENSION FUNCTIONS OF SUBSETS OF $\mathbb{R}^{d}$
}

\author{
L. OLSEN \\ Department of Mathematics, University of St Andrews, \\ St Andrews, Fife KY16 9SS, UK (lo@st-and.ac.uk)
}

(Received 18 September 2003)

Abstract For a subset $E \subseteq \mathbb{R}^{d}$ and $x \in \mathbb{R}^{d}$, the local Hausdorff dimension function of $E$ at $x$ is defined by

$$
\operatorname{dim}_{\mathrm{loc}}(x, E)=\lim _{r \searrow 0} \operatorname{dim}(E \cap B(x, r)),
$$

where 'dim' denotes the Hausdorff dimension. Using some of our earlier results on so-called multifractal divergence points we give a short proof of the following result: any continuous function $f: \mathbb{R}^{d} \rightarrow[0, d]$ is the local dimension function of some set $E \subset \mathbb{R}^{d}$. In fact, our result also provides information about the rate at which the $\operatorname{dimension} \operatorname{dim}(E \cap B(x, r))$ converges to $f(x)$ as $r \searrow 0$.

Keywords: Hausdorff dimension; normal numbers; non-normal numbers; frequencies of digits; divergence points

2000 Mathematics subject classification: Primary 28A80

\section{Introduction and statement of results}

For a subset $E \subseteq \mathbb{R}^{d}$ and $x \in \mathbb{R}^{d}$, we define the local Hausdorff dimension function of $E$ at $x$ by

$$
\operatorname{dim}_{\mathrm{loc}}(x, E)=\lim _{r \searrow 0} \operatorname{dim}(E \cap B(x, r)),
$$

where 'dim' denotes the Hausdorff dimension. The local packing dimension function of $E$ at $x$ is defined similarly, i.e. by

$$
\operatorname{Dim}_{\mathrm{loc}}(x, E)=\lim _{r \searrow 0} \operatorname{Dim}(E \cap B(x, r)),
$$

where 'Dim' denotes the packing dimension. The reader is referred to [2] for the definitions of the Hausdorff and the packing dimensions. The local Hausdorff dimension function of a set has recently found several applications in fractal geometry and information theory $($ cf. $[\mathbf{3}, \mathbf{8}])$. The purpose of this paper is to apply the results from $[\mathbf{4}-\mathbf{7}]$ on socalled multifractal divergence points to give a short proof of the fact that any continuous function $f: \mathbb{R}^{d} \rightarrow[0, d]$ is the local dimension function of some set $E \subseteq \mathbb{R}^{d}$. In fact, our result also provides information about the rate at which the dimensions $\operatorname{dim}(E \cap B(x, r))$ 
and $\operatorname{Dim}(E \cap B(x, r))$ converge to $f(x)$ as $r \searrow 0$ (see (1.1) below). For an arbitrary function $f: \mathbb{R}^{d} \rightarrow \mathbb{R}$ and $x \in \mathbb{R}^{d}$, we let

$$
\omega_{f}(x, r)=\sup _{x_{1}, x_{2} \in B(x, r)}\left|f\left(x_{1}\right)-f\left(x_{2}\right)\right|
$$

denote the modulus of continuity of $f$ at $x$, and observe that $f$ is continuous at $x$ if and only if $\omega_{f}(x, r) \rightarrow 0$ as $r \searrow 0$.

Theorem 1.1. Let $f: \mathbb{R}^{d} \rightarrow[0, d]$ be an arbitrary function. Then there exists a set $E \subseteq \mathbb{R}^{d}$ such that

$$
\left.\begin{array}{r}
|f(x)-\operatorname{dim}(E \cap B(x, r))| \leqslant \omega_{f}(x, r), \\
|f(x)-\operatorname{Dim}(E \cap B(x, r))| \leqslant \omega_{f}(x, r),
\end{array}\right\}
$$

for all $x \in \mathbb{R}^{d}$ and all $r>0$. In particular, if $f$ is continuous, then

$$
f(x)=\operatorname{dim}_{\mathrm{loc}}(x, E)=\operatorname{Dim}_{\mathrm{loc}}(x, E)
$$

for all $x \in \mathbb{R}^{d}$.

It is not difficult to construct discontinuous local dimension functions. Indeed, let $C \subseteq$ $\mathbb{R}$ denote the classical ternary Cantor set. If we define $f: \mathbb{R} \rightarrow[0,1]$ by $f(x)=\operatorname{dim} C=$ $\log 2 / \log 3$ for $x \in C$ and $f(x)=0$ for $x \notin C$, then clearly $f(x)=\operatorname{dim}_{\mathrm{loc}}(x, C)$ for all $x \in \mathbb{R}$. On the other hand, there exist very simple discontinuous functions $f: \mathbb{R} \rightarrow[0,1]$ that are not local dimension functions of any sets $E \subseteq \mathbb{R}$. For example, the function $f: \mathbb{R} \rightarrow[0,1]$ defined by $f(x)=0$ for $x \neq 0$ and $f(0)=1$ is easily seen not to be the local dimension function of any set $E \subseteq \mathbb{R}$. This observation leads to the following question.

Question 1.2. Find a characterization of those functions $f: \mathbb{R}^{d} \rightarrow[0, d]$ for which there exists a set $E \subseteq \mathbb{R}^{d}$ such that $f(x)=\operatorname{dim}_{\text {loc }}(x, E)$ for all $x \in \mathbb{R}^{d}$.

The proof of Theorem 1.1 is given in $\S 2$. However, before proving Theorem 1.1, we will indicate why the theory of divergence points is essential in the proof of Theorem 1.1. For $x \in \mathbb{R}$, let $[x]$ denote the integer part of $x$ and write

$$
x=[x]+\sum_{n=1}^{\infty} \frac{d_{n}(x)}{2^{n}}
$$

for the unique non-terminating binary expansion of $x$. For a digit $j \in\{0,1\}$ and a positive integer $n$ write

$$
\Pi_{j}(x ; n)=\frac{\left|\left\{1 \leqslant i \leqslant n \mid d_{i}(x)=j\right\}\right|}{n}
$$

for the frequency of the digit $j$ among the first $n$ binary digits of $x$. A real number $x$ is called a divergence point if there exists at least one digit $j$ such that the limiting frequency $\lim _{n} \Pi_{j}(x ; n)$ does not exist, and multifractal analysis of divergence points 
refers to the study of the Hausdorff and packing dimensions of various sets of divergence points. For $t \geqslant 0$, we write

$$
\Delta(t)=\left\{\left(x_{k}\right)_{k} \in \mathbb{R}^{d} \mid \lim _{n} \Pi_{0}\left(x_{k} ; n\right)=t \text { for all } k\right\} .
$$

Next, let $h:[0,1] \rightarrow[0, d]$ denote the (entropy) function

$$
h(t)=-\frac{d}{\log 2}(t \log t+(1-t) \log (1-t)) .
$$

Observe that $h$ is strictly increasing on $\left[0, \frac{1}{2}\right]$, and therefore has an inverse $h^{-1}:[0, d] \rightarrow$ $\left[0, \frac{1}{2}\right]$. For a given function $f: \mathbb{R}^{d} \rightarrow[0, d]$, we now define the set $E$ by

$$
E=\bigcup_{0 \leqslant t \leqslant d}\left(\Delta\left(h^{-1}(t)\right) \cap\{t \leqslant f\}\right),
$$

and claim that $|f(x)-\operatorname{dim}(E \cap B(x, r))| \leqslant \omega_{f}(x, r)$ and $|f(x)-\operatorname{Dim}(E \cap B(x, r))| \leqslant$ $\omega_{f}(x, r)$ for all $x \in \mathbb{R}^{d}$ and all $r>0$. To prove this claim we must show that $m(x, r) \leqslant$ $\operatorname{dim}(E \cap B(x, r))$ and that $\operatorname{Dim}(E \cap B(x, r)) \leqslant M(x, r)$, where $m(x, r)=\inf _{y \in B(x, r)} f(y)$ and $M(x, r)=\sup _{y \in B(x, r)} f(y)$. The first inequality is proved in $\S 2$ without any reference to divergence points. However, the theory of divergence points play a crucial role in the proof of the second inequality. Indeed, we first show that (the details are given in $\S 2$ )

$$
\operatorname{Dim}(E \cap B(x, r)) \leqslant \operatorname{Dim}\left(\bigcup_{0 \leqslant t \leqslant M(x, r)} \Delta\left(h^{-1}(t)\right)\right) .
$$

Also, it follows without too much effort from (the packing dimension version of) a classical result due the Besicovith and Eggleston (cf. [1, p. 139]) that Dim $\Delta(t)=h(t)$ (the details are given in $\S 2$ ), whence

$$
\sup _{0 \leqslant t \leqslant M(x, r)} \operatorname{Dim} \Delta\left(h^{-1}(t)\right)=\sup _{0 \leqslant t \leqslant M(x, r)} h\left(h^{-1}(t)\right)=M(x, r) .
$$

Ideally, we would now like to combine (1.4) and (1.5) to obtain the following string of (in)equalities,

$$
\begin{aligned}
\operatorname{Dim}(E \cap B(x, r)) & \leqslant \operatorname{Dim}\left(\bigcup_{0 \leqslant t \leqslant M(x, r)} \Delta\left(h^{-1}(t)\right)\right) \\
& =\sup _{0 \leqslant t \leqslant M(x, r)} \operatorname{Dim} \Delta\left(h^{-1}(t)\right) \\
& =M(x, r) .
\end{aligned}
$$

However, the argument leading from (1.4) and (1.5) to (1.6) requires that the following equality holds for all $u \geqslant 0$ :

$$
\operatorname{Dim}\left(\bigcup_{0 \leqslant t \leqslant u} \Delta(t)\right)=\sup _{0 \leqslant t \leqslant u} \operatorname{Dim} \Delta(t) .
$$


Unfortunately, since the packing dimension is not uncountable stable, it is not clear that equality (1.7) holds. The key result that allows us to prove (1.7) is based on the methods and techniques from multifractal analysis of divergence points developed in [4-7]. The details of this argument are presented in $\S 2$.

\section{Proof of Theorem 1.1}

Theorem 2.1 below is proved in [5] using methods and techniques from $[\mathbf{4}, \mathbf{6}, \mathbf{7}]$ on multifractal divergence points, and gives the Hausdorff and packing dimensions of some sets of $d$-tuples of numbers defined by their $N$-adic expansion. Theorem 2.1 is the key result needed to justify equality (1.7) in the proof of Theorem 1.1.

We first introduce some notation. Fix a positive integer $N \geqslant 2$. For $x \in \mathbb{R}$, recall that $[x]$ denotes the integer part of $x$ and write

$$
x=[x]+\sum_{n=1}^{\infty} \frac{d_{N, n}(x)}{N^{n}}
$$

for the unique non-terminating $N$-adic expansion of $x$. For a digit $j \in\{0,1, \ldots, N-1\}$ and a positive integer $n$ write

$$
\Pi_{N, j}(x ; n)=\frac{\left|\left\{1 \leqslant i \leqslant n \mid d_{N, i}(x)=j\right\}\right|}{n}
$$

for the frequency of the digit $j$ among the first $n$ of the $N$-adic digits of $x$. Also, let

$$
\Delta_{N}=\left\{\left(p_{j}\right)_{j=0,1, \ldots, N-1} \mid p_{j} \geqslant 0, \sum_{j} p_{j}=1\right\}
$$

denote the family of probability vectors in $\mathbb{R}^{N}$, and define $H: \Delta_{N} \rightarrow \mathbb{R}$ by

$$
H(\boldsymbol{p})=-\frac{\sum_{j} p_{j} \log p_{j}}{\log N}
$$

for $\boldsymbol{p}=\left(p_{j}\right)_{j} \in \Delta_{N}$. Finally, for a sequence $\left(x_{n}\right)_{n}$ in a metric space $X$, we let $\mathrm{A}\left(x_{n}\right)$ denote the set of accumulation points of the sequence $\left(x_{n}\right)_{n}$, i.e.

$\mathrm{A}\left(x_{n}\right)=\left\{x \in X \mid\right.$ there exists a subsequence $\left(x_{n_{k}}\right)_{k}$ such that $\left.x_{n_{k}} \rightarrow x\right\}$.

Theorem 2.1. Let $\Lambda \subseteq\{1, \ldots, d\} \times\{0,1, \ldots, N-1\}$ and write

$$
\Gamma=\left\{\left(p_{k, j}\right)_{(k, j) \in \Lambda} \mid p_{k, j} \geqslant 0, \sum_{\substack{j \\(k, j) \in \Lambda}} p_{k, j} \leqslant 1 \text { for all } k\right\} .
$$

For $\boldsymbol{p}=\left(p_{k, j}\right)_{(k, j) \in \Lambda} \in \Gamma$ define $\boldsymbol{u}_{k}(\boldsymbol{p})=\left(\boldsymbol{u}_{k, j}(\boldsymbol{p})\right)_{j} \in \Delta_{N}$ by

$$
\boldsymbol{u}_{k, j}(\boldsymbol{p})= \begin{cases}p_{k, j}, & \text { for }(k, j) \in \Lambda, \\ \left(1-\sum_{\substack{i \\(k, i) \in \Lambda}} p_{k, i}\right) / \sum_{\substack{i \\(k, i) \notin \Lambda}} 1, & \text { for }(k, j) \notin \Lambda .\end{cases}
$$


If $C \subseteq \Gamma$ is closed and convex, then

$$
\begin{aligned}
\operatorname{dim}\left\{\left(x_{k}\right)_{k} \in \mathbb{R}^{d} \mid \mathrm{A}\left(\left(\Pi_{N, j}\left(x_{k} ; n\right)\right)_{(k, j) \in \Lambda}\right) \subseteq C\right\} & \\
& =\operatorname{Dim}\left\{\left(x_{k}\right)_{k} \in \mathbb{R}^{d} \mid \mathrm{A}\left(\left(\Pi_{N, j}\left(x_{k} ; n\right)\right)_{(k, j) \in \Lambda}\right) \subseteq C\right\} \\
& =\sup _{\boldsymbol{p} \in C}\left(H\left(\boldsymbol{u}_{1}(\boldsymbol{p})\right)+\cdots+H\left(\boldsymbol{u}_{d}(\boldsymbol{p})\right)\right) .
\end{aligned}
$$

Proof of Theorem 1.1. We can now prove Theorem 1.1. Let the sets $\Delta(t)$ and $E$ be defined as in (1.2) and (1.3), respectively. Recall that

$$
\begin{aligned}
& m(x, r)=\inf _{y \in B(x, r)} f(y), \\
& M(x, r)=\sup _{y \in B(x, r)} f(y),
\end{aligned}
$$

for $x \in \mathbb{R}^{d}$ and $r>0$.

Claim 2.2. We have

$$
\operatorname{Dim}(E \cap B(x, r)) \leqslant M(x, r) .
$$

Proof of Claim 2.2. Write $I=\left[0, h^{-1}(M(x, r))\right]$. First observe that

$$
\begin{aligned}
E \cap B(x, r) & =\bigcup_{0 \leqslant t \leqslant d}\left(\Delta\left(h^{-1}(t)\right) \cap\{t \leqslant f\}\right) \cap B(x, r) \\
& =\bigcup_{0 \leqslant t \leqslant M(x, r)}\left(\Delta\left(h^{-1}(t)\right) \cap\{t \leqslant f\}\right) \cap B(x, r) \\
& \subseteq \bigcup_{0 \leqslant t \leqslant M(x, r)} \Delta\left(h^{-1}(t)\right) \\
& \subseteq\left\{\left(x_{k}\right)_{k} \in \mathbb{R}^{d} \mid \mathrm{A}\left(\left(\Pi_{0}\left(x_{k} ; n\right)\right)_{k}\right) \subseteq I^{d}\right\} .
\end{aligned}
$$

Hence

$$
\operatorname{Dim}(E \cap B(x, r)) \leqslant \operatorname{Dim}\left\{\left(x_{k}\right)_{k} \in \mathbb{R}^{d} \mid \mathrm{A}\left(\left(\Pi_{0}\left(x_{k} ; n\right)\right)_{k}\right) \subseteq I^{d}\right\} .
$$

Next, observe that Theorem 2.1 with $N=2, \Lambda=\{(1,0), \ldots,(d, 0)\}$ and $C=I^{d}$ implies that

$$
\begin{aligned}
\operatorname{Dim}\left\{\left(x_{k}\right)_{k} \in \mathbb{R}^{d} \mid \mathrm{A}\left(\left(\Pi_{0}\left(x_{k} ; n\right)\right)_{k}\right) \subseteq I^{d}\right\} & \leqslant \sup _{\left(p_{k}\right)_{k} \in I^{d}}\left(H\left(p_{1}, 1-p_{1}\right)+\cdots+H\left(p_{d}, 1-p_{d}\right)\right) \\
& =\sup _{\left(p_{k}\right)_{k} \in I^{d}} \frac{1}{d}\left(h\left(p_{1}\right)+\cdots+h\left(p_{d}\right)\right) \\
& =\frac{1}{d}\left(h\left(h^{-1}(M(x, r))\right)+\cdots+h\left(h^{-1}(M(x, r))\right)\right) \\
& =M(x, r) .
\end{aligned}
$$

Combining (2.1) and (2.2) completes the proof of Claim 2.2. 
Claim 2.3. We have

$$
m(x, r) \leqslant \operatorname{dim}(E \cap B(x, r)) .
$$

Proof of Claim 2.3. We clearly have

$$
\begin{aligned}
\operatorname{dim}(E \cap B(x, r)) & \geqslant \operatorname{dim}\left(\Delta\left(h^{-1}(m(x, r))\right) \cap\{m(x, r) \leqslant f\} \cap B(x, r)\right) \\
& =\operatorname{dim}\left(\Delta\left(h^{-1}(m(x, r))\right) \cap B(x, r)\right) .
\end{aligned}
$$

Next, observe that whether or not a number $x$ belongs to $\Delta(t)$ only depends on the limiting behaviour of the $N$-adic digits of $x$. It follows from this observation that $\operatorname{dim}(\Delta(t) \cap B(x, r))=\operatorname{dim} \Delta(t)$. We therefore conclude from (2.3) that

$$
\operatorname{dim}(E \cap B(x, r)) \geqslant \operatorname{dim} \Delta\left(h^{-1}(m(x, r))\right) .
$$

Again, observe that Theorem 2.1 with $N=2, \Lambda=\{(1,0), \ldots,(d, 0)\}$ and $C=\{(t, \ldots, t)\}$ implies that

$$
\begin{aligned}
\operatorname{dim} \Delta(t) & =\sup _{\left(p_{k}\right)_{k} \in\{(t, \ldots, t)\}}\left(H\left(p_{1}, 1-p_{1}\right)+\cdots+H\left(p_{d}, 1-p_{d}\right)\right) \\
& =\frac{1}{d}(h(t)+\cdots+h(t)) \\
& =h(t) .
\end{aligned}
$$

Finally, combining (2.4) and (2.5) yields

$$
\operatorname{dim}(E \cap B(x, r)) \geqslant h\left(h^{-1}(m(x, r))\right)=m(x, r) .
$$

This completes the proof of Claim 2.3.

Since $\operatorname{dim} A \leqslant \operatorname{Dim} A$ for all $A \subseteq \mathbb{R}^{d}$, Theorem 1.1 follows immediately from Claim 2.2 and Claim 2.3. This completes the proof of Theorem 1.1.

\section{References}

1. P. Billingsley, Ergodic theory and information (Wiley, 1965).

2. K. J. FALCONER, Fractal geometry-mathematical foundations and applications (Wiley, 1990).

3. H. JÜrgensen and L. Staiger, Local Hausdorff dimension, Acta Informatica 32 (1995), 491-507.

4. L. OLSEN, Multifractal analysis of divergence points of deformed measure theoretical Birkhoff averages, J. Math. Pures Appl. 82 (2003), 1591-1649.

5. L. Olsen, Applications of multifractal divergence points to some sets of $d$-tuples of numbers defined by their $N$-adic expansion, Bull. Sci. Math. 128 (2004), 265-289.

6. L. OlSEN AND S. WinteR, Multifractal analysis of divergence points of deformed measure theoretical Birkhoff averages, II, preprint (2001).

7. L. OLSEN AND S. WinteR, Normal and non-normal points of self-similar sets and divergence points of self-similar measures, J. Lond. Math. Soc. 67 (2003), 103-122.

8. T. Rushing, Hausdorff dimension of wild fractals, Trans. Am. Math. Soc. 334 (1992), 597-613. 